\section{An ex vivo study on immunohistochemical localization of MMP-7 and MMP-9 in temporomandibular joint discs with internal derangement}

\author{
C. Loreto, ${ }^{1}$ R. Leonardi, ${ }^{2}$ G. Musumeci, \\ G. Pannone, ${ }^{3}$ S. Castorina ${ }^{1}$ \\ 'Department of Bio-Medical Sciences, \\ Anatomy Section, University of Catania, \\ Italy; ${ }^{2}$ Department of Medical-Surgical \\ Sciences, Dentistry Section, University \\ of Catania, Italy; ${ }^{3}$ Department of Surgical \\ Sciences, Institute of Pathology and \\ Cytopathology, University of Foggia, Italy
}

\section{Abstract}

Internal derangement (ID) is among the most common disorders of the temporomandibular joint (TMJ). Previous research by our group highlighted a correlation between apoptosis and TMJ ID. Metalloproteinases (MMP)-7 and -9 have been shown to play an important role in extracellular matrix ECM) homeostasis and, through it, in joint disc remodelling. The immunohistochemical expression of MMP-7 and -9 was investigated in discs from patients with TMJ ID and from healthy donors and compared with the degree of histological tissue degeneration. The collagen fibre arrangement in pathological discs exhibited varying degrees of disruption. New vessels were consistently detected; endothelial cells from these vessels were immunolabelled with both MMP-7 and MMP-9. More or less intense MMP-7 and MMP-9 immunolabelling was detected in the cytoplasm of disc cells from all patients. MMP-7 and MMP-9 immunostaining was significantly different between pathological and normal discs and correlated with the extent of histopathological degeneration. MMP-7 and MMP-9 upregulation in discs from patients with TMJ ID demonstrates their involvement in disc damage in this disorder. A greater understanding of these processes could help identify ways to curb MMP overproduction without affecting their tissue remodelling action. The design of specific inhibitors for these MMPs would not only help to gain insights into the biological roles of MMPs, but would also aid in developing therapeutic interventions for diseases associated with abnormal ECM degradation.

\section{Introduction}

Internal derangement (ID) is among the most common disorders of the temporomandibular joint (TMJ). Derangement, a clinical feature used to classify TMJ disorders (TMDs), is also used to indicate an abnormal relationship of the articular disc to the mandibular condyle and the articular eminence. TMJ disc displacement is followed by progressive degradation of the extracellular matrix (ECM) of its connective tissue and by histopathological changes that eventually lead to permanent loss of function. ${ }^{1}$ Joint changes begin to arise in the posterior disc band, ${ }^{2}$ based on the type and extent of disc displacement; advanced ID corresponds to a degenerated disc configuration. ${ }^{3}$

Normal TMJ disc contains ECM macromolecules, i.e. collagen, glycosaminoglycans, proteoglycans and other molecules related to joint disc lubrication..$^{4-5}$ The collagen types identified in this tissue to date are I, II, III, VI, IX, and XII. TMJ disc collagen is almost entirely type I with trace amounts of types II and III. ${ }^{6}$ In healthy TMJ disc the balance between ECM synthesis and degradation results in a state of dynamic equilibrium. Pathological disc changes can therefore be the consequence of disruptions of this balance favouring proteolysis. The main molecules involved in ECM degradation are metalloproteinases (MPs) and their inhibitors.

Matrix metalloproteinases (MMPs), collectively called matrixins, participate in ECM degradation. In physiological conditions their activities are precisely regulated at the level of transcription, activation of the precursor zymogens, interaction with specific ECM components, and inhibition by endogenous inhibitors. Disruption of this control may result in diseases of the joints as well as of the orofacial region. ${ }^{7-12}$ Animal and human studies both indicate that at least collagenases MMP$1,-8$, and -13 , gelatinases MMP-2 and -9 , stromelysin MMP-3, and matrilysin MMP-7 are involved in different stages of joint disease pathogenesis. Increased concentrations of MMP-1,-2,-3 and -9 have been detected in TMJ synovial fluid of patients with IDs and/or osteoarthritis (OA). ${ }^{13-16}$ Information regarding MMP-9 expression is scarce, at least at the protein level, nor have any data been reported on MMP-7. Exhaustive information on MMP immunohistochemical expression in TMJ disc tissue is available only for MMP-13. ${ }^{17}$ MMP-7 (matrilysin 1) processes ECM components as well as cell surface molecules such as pro- $\alpha$ defensin, Fas-ligand, pro-tumour necrosis factor (TNF)- $\alpha$, and E-cadherin. MMP-9, a gelatinase (gelatinase B), digests denatured collagens.
Correspondence: Dr. Carla Loreto, Department of Bio-Medical Sciences, Anatomy Section, University of Catania, via S. Sofia 87, 95123 Catania, Italy.

Tel. +39.095 .3782038 - Fax: +39.095 .3782046 .

E-mail: carla.loreto@unict.it

Key words: MMP-7, MMP-9 temporomandibular joint disc, internal derangement.

Received for publication: 4 November 2012. Accepted for publication: 4 February 2013.

This work is licensed under a Creative Commons Attribution NonCommercial 3.0 License (CC BYNC 3.0).

(C) Copyright C. Loreto et al., 2013

Licensee PAGEPress, Italy

European Journal of Histochemistry 2013; 57:e12 doi:10.4081/ejh.2013.e12

MMPs also have a role in cell proliferation, migration, differentiation, and apoptosis and in angiogenesis. ${ }^{18}$ ECM degradation by MMP-9 promotes cell death. ${ }^{19}$ Previous research by our group has highlighted a correlation between apoptosis and TMJ ID, ${ }^{20-24}$ and an important role for MMP-9 and 7 in ECM homeostasis.

The aim of this immunohistochemical study was to investigate their expression in TMJ discs with ID and compare the immunohistochemical data to the degree of tissue degeneration.

\section{Materials and Methods}

\section{Patients and tissues}

Twenty-five paraffin blocks of displaced TMJ disc specimens from earlier investigations were retrieved from the archives of the University of Catania (Italy). ${ }^{25-27}$ Their use had already been approved by the relevant ethics committees; similarly, the patients' informed consent had been obtained before tissue collection. The discs had been removed from 16 females and 9 males with TMJ ID, 11 with anterior disc displacement with reduction (ADDwR) and 14 with anterior disc displacement without reduction (ADDwoR) diagnosed on the basis of history, clinical examination, and magnetic resonance imaging data. Mean patient age at the time of surgery was $34.2 \pm 5.4$ years; mean disease duration from ID symptom onset to surgery was $8.7 \pm 1.2$ months. Unassisted maximum mouth opening (MM0) and a visual analogue scale (VAS) for pain were used to assess disease severity. MM0 was measured with a millimetre ruler as interincisal distance; pain intensity in the preceding week was rated by patients on a $100 \mathrm{~mm}$ VAS 
from 0 (no pain) to 100 (the worst imaginable pain). The diagnosis that led to disc excision was ID with pain and functional impairment. The inclusion criteria for this study were: i) unsuccessful conservative management; ii) tenderness to TMJ palpation; and iii) TMJ pain or interference with jaw movement; exclusion criteria were: i) other TMJ diseases; ii) dentofacial deformity; iii) major jaw trauma; iv) previous TMJ surgery; and v) prior TMJ treatment with steroid injections. The discs removed from the 25 patients were macroscopically deformed and none had preserved a normal biconcave shape. The anterior, intermediate and posterior band were preserved in all specimens. Four TMJ discs from the collection of Catania University's Anatomy Institute were also studied. They were autopsy specimens from one male and three female donors (mean age $49.7 \pm 4.4$ years) which were selected for their virtually normal shape and condition, since none had macroscopic signs of degenerative or inflammatory joint disease on dissection, nor were they displaced; in addition the donors' clinical histories were negative for generalized joint disease or TMJ arthropathy.

\section{Histology}

The TMJ discs were explanted, cleaned of adhering tissues, and fixed in $10 \%$ buffered formalin for $24 \mathrm{~h}$. They were processed as described previously. ${ }^{28}$ Briefly tissues were dehydrated using the following sequence: $50 \%$ $\mathrm{EtOH} \times 1 \mathrm{~h}, 70 \% \mathrm{EtOH} \times 1 \mathrm{~h}, 80 \% \mathrm{EtOH} \times 1.5$ h, $95 \% \mathrm{EtOH} \times 12$ h, $100 \% \mathrm{EtOH} \times 1$ h-repeat twice, xylene $\times 1 \mathrm{~h}$, placed in a metal mold and embedded in paraffin at $60^{\circ} \mathrm{C}$ overnight. Section of $5 \mu \mathrm{m}$ were cut and stained with haematoxylin for routine morphological evaluation. Disc sections were observed with an Axioplan Zeiss light microscope (Jena Germany).

\section{Immunohistochemistry}

Sections were processed as previously described. ${ }^{29,30}$ Briefly, they were incubated for 30 min in $0.3 \% \mathrm{H}_{2} \mathrm{O}_{2}$ /methanol to quench endogenous peroxidase activity then rinsed for $20 \mathrm{~min}$ with phosphate-buffered saline (PBS; BioOptica, Milan, Italy). The sections were irradiated as previously described. Then, half of the sections of each sample were incubated in rabbit polyclonal anti-MMP-9 (NB-P1-72189) and the other half in anti MMP-7 antibody (NB-300 1000; both from Novus Biologicals, Littleton, C0, USA) diluted 1:100 in PBS, 0.1\% BSA, and incubated overnight at $4^{\circ} \mathrm{C}$. The secondary antibody, biotinylated anti-mouse/anti-rabbit IgG, was applied for $30 \mathrm{~min}$ at room temperature followed by the avidin-biotin-peroxidase complex (Vector Elite Kit Abbott, Chicago, IL, USA). The immunoreaction was visualized by incubating sections in a $0.1 \% 3,3$ '-diaminobenzidine and $0.02 \%$ hydrogen peroxide solution (DAB substrate kit, Vector Laboratories, Burlingame, CA, USA) for $4 \mathrm{~min}$. Finally sections were lightly counterstained with Mayer's haematoxylin (Histolab Products AB, Goteborg, Sweden) and mounted on GVA mount (Zymed Laboratories, San Francisco, CA, USA).

MPP-7 and MMP-9 staining intensity and the proportion of immunopositive cells found in each of the three areas of the disc (anterior, intermediate zone and posterior) were examined in blind by light microscopy and recorded by three anatomists. The three disc areas, anterior, intermediate and posterior band, were examined. Intensity of staining (IS) was graded from 0 to 4 as: $0=$ no detectable staining, 1 = weak, 2 = moderate, 3 =strong, $4=$ very strong. Cells immunopositive for MPP-7 and MMP-9 [extent score (ES)] were expressed as a percentage of the final number of 100 cells at $200 x$ magnification and classed separately by the three anatomists into four categories: $0=<5 \% ; 1=6-30 \% ; 2=31-50 \%$; $3=>50 \%$, and $4=>75 \%$. The final staining score (FSS) represented the sum of IS and ES. The FSS obtained for each of the 3 disc areas were added and divided by 3 to calculate the mean ( \pm standard deviation) FSS for each disc. Positive and negative controls were performed as previously described. ${ }^{31} \mathrm{~A}$ disc histopathological degeneration score was calculated for each disc as described previously. ${ }^{32}$

\section{Statistical analysis}

The expression level of MMP-7 and MMP-9 was compared with the histopathological degeneration score using Spearman's test. Interobserver agreement was expressed as kappa coefficient. The anterior, intermediate and posterior bands were compared in ADDwR and ADDwoR patients. All data were analysed with the SPSS program (SPSS $®$ release 16.0, Chicago, IL, USA).

\section{Results}

\section{Histology}

Abnormal collagen fibre arrangement and fragmentation and tearing of collagen bundles were demonstrated in patients' haematoxylinstained discs, whereas multidirectional collagen bundles were preserved in control discs. Patients' discs also showed reduced cellularity and an altered cell population ratio, with an increased number of chondrocyte-like cells related to the severity of the morphological disc damage, and a decreased number of fibroblast-like cells, particularly in ADDwR discs.

\section{Immunohistochemistry}

The TMJ ID patients had received a variety of unsuccessful non-surgical treatments for at least 6 months prior to surgery. Histological examination of haematoxylin-stained discs demonstrated in all specimens different degrees of disruption of the collagen fibre arrangement that ranged from fragmentation to tears and splitting. New vessels were always detected. The mean histopathological score of the 25 discs was $4.27 \pm 1.76$ (range $3-7$ ). Multidirectional collagen bundles were preserved in control discs, which also showed an absence of new vessels and of signs of nonspecific tissue degeneration.

More or less intense MMP-7 and MMP-9 immunolabelling was detected in the cytoplasm of disc cells from all patients (Figures 1, 2 and 3). The mean FSS of the pathological discs was $2.27 \pm 0.71$ (range 1.6-3.9) for MMP-7 and 2.87 \pm 0.92 (range 1.6-3.7) for MMP-9. The control discs exhibited faint immunostaining (MMP-7, 0.65 \pm 0.41 and MMP-9, 0.45 \pm 0.75 ). A greater proportion of cells positive for MMP-7 and MMP-9 was documented in ADDwR and ADDwo R sections than in normal disc. Immunopositivity also varied in the different disc areas; in particular, labelled cells were significantly more numerous $(\mathrm{P}<0.01)$ in the posterior band compared with the anterior and intermediate bands in ADDwoR discs. In addition, a significantly greater proportion of labelled cells was seen in the anterior $(+)$ and intermediate $(++)$ band of ADDwR compared with ADDwoR discs (both bands, $\mathrm{P}<0.05$ ). No difference among disc regions was found in normal specimens. MMP-7 was also detected in synovium, chiefly in cells; very small amounts were bound to the ECM, indicating its synthesis. Intracellular MMP-7 staining was focal, with marked regional variations, but the extent of staining generally correlated $(\mathrm{P} \leq 0.01)$ with the degree of synovial inflammation. Endothelial cells from newly-formed vessels were immunolabelled with both MMP7 and MMP-9. The kappa coefficient was 0.89 (nearly perfect agreement). The difference in MMP-7 and MMP-9 immunostaining between pathological and normal discs was significant $(\mathrm{P}=001)$ and correlated with the histopathological degeneration score (Spearman's rho $=0.639$ for MMP-7 and $=0.529$ for MMP-9).

\section{Discussion}

Degenerative changes alter the physical and functional properties of the TMJ, leading to an inability to withstand loading stress that eventually becomes irreversible. ${ }^{33,34}$ Clinically mild TMJ ID is characterized by disc displacement with or without bone remodelling, whereas 
severe ID involves disc or disc attachment perforation, bone remodelling, and osteoarthritic changes. The different proportions of cells staining for MMP-7 and MMP-9 documented in the three regions of pathological compared with normal discs are explained by the site where the maximum compressive stress is exerted. Disc displacement during mouth opening entails that in pathological disc this stress is exerted on the posterior band. Moreover different immunolabelling patterns were also found in ADDwR and ADDwoR discs. In the former discs maximum stress is exerted on the anterior band before disc recapture; ${ }^{35}$ when the disc is reduced, the maximum stress is exerted on the intermediate zone, where caspase 3-positive cells were indeed most numerous. In ADDwoR discs maximum compressive stress is exerted on the posterior band, ${ }^{35}$ where we detected a greater expression of caspase- 3 compared with the other two bands. These pathophysiological considerations contribute to explain the finding of different proportions of cells positive for MMP-7 and MMP-9 in posterior, intermediate and anterior disc bands. Different types of collagen and non-collagenous proteins as well as MMPs seem to be involved in disc and joint changes, at least as demonstrated by examination of synovial fluid from patients with TMDs. However, even though immunohistochemical findings provide critical clinical information, ${ }^{36,37}$ very little work has been done on TMJ disc MMPs because disc tissue is difficult to obtain.

The present study demonstrates for the first time that MMP-7 and MMP-9 are upregulated in discs from patients with TMJ ID and provides evidence of a role for them in disc damage in this disorder. Interestingly, MMP-7 and MMP-9 are expressed in arthritic joints and can degrade a number of matrix proteins in

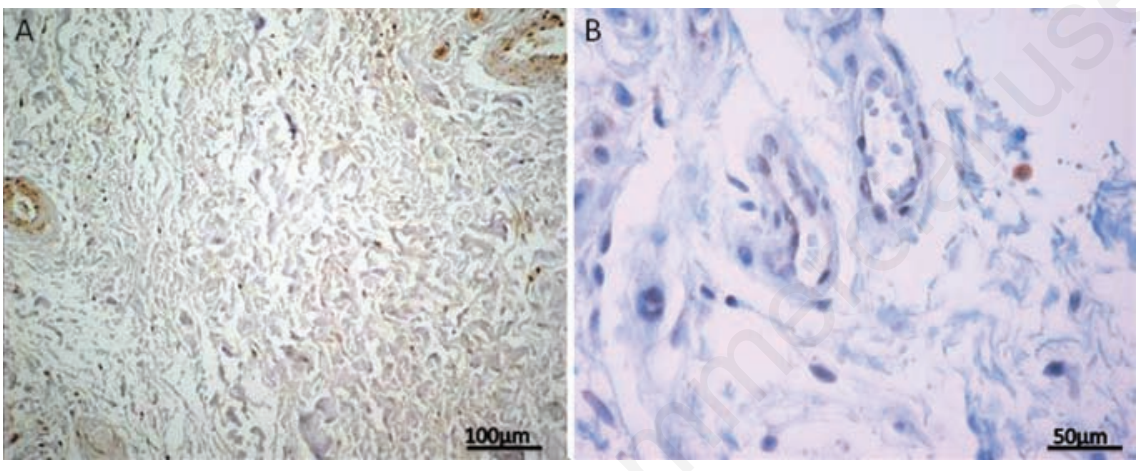

Figure 1. A) MMP-7 immunoexpression in fibroblast-like and chondrocyte-like cells in the posterior band of the TMJ ID disc from a patient with ADDwoR; newly-formed vessels are also immunolabelled; magnification: 20x; scale bar: $100 \mu \mathrm{m}$. B) Higher magnification of the posterior band of the TMJ ID disc from a patient with ADDwoR shows MMP-7 localization in endothelial cells from newly-formed vessels; magnification: 40x; scale bar: $50 \mu \mathrm{m}$.

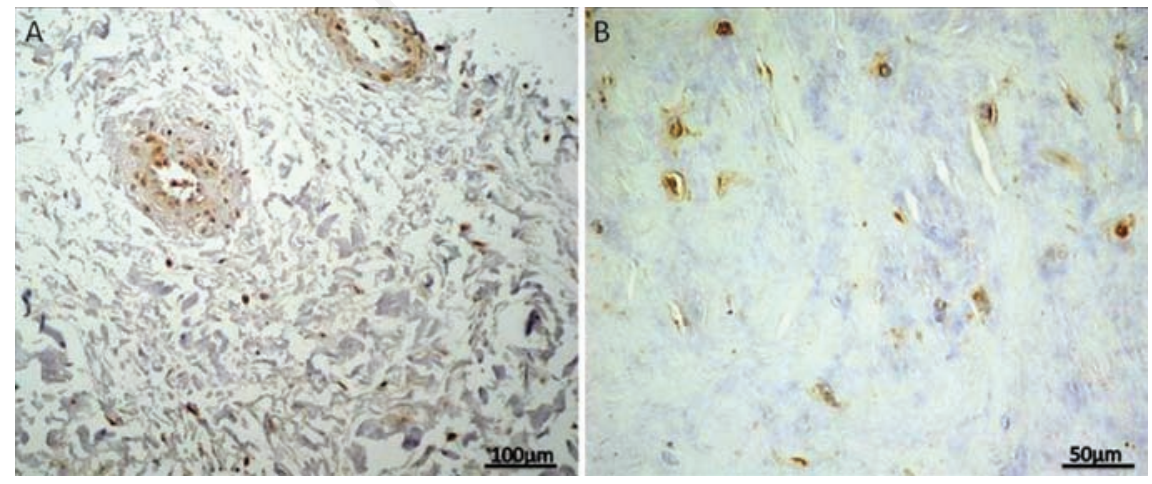

Figure 2. A) MMP-9 immunostaining in fibroblast-like and chondrocyte-like cells of the posterior band of the TMJ ID disc from a patient with ADDwoR; magnification: 20x; scale bar: $100 \mu \mathrm{m}$. B) Higher magnification of the posterior band of the TMJ ID disc from a patient with $A D D w o R$ with chondrocyte-like cells immunolabbeled with MMP9; magnification: 40x; scale bar: $50 \mu \mathrm{m}$. the joint. ${ }^{38-42}$ Furthermore they are involved in new blood vessel formation through cleaving of plasminogen and generation of angiostatin molecules; ${ }^{43}$ these findings may partly account for the newly formed vessels found in pathological TMJ discs. ${ }^{33}$ Increased immunoreactivity for elastolytic proteases, particularly MMP-7 and MMP-9, has also been demonstrated in areas of elastin depletion in floppy eyelid syndrome specimens compared with controls. ${ }^{43}$ Unexpectedly, elastic system fibres (oxytalan, elaunin, and elastic fibres) have been described in severely damaged TMJ disc, ${ }^{44}$ where they appear to ensure biomechanical resistance to stretch and compression by reinforcing regions lacking collagen bundles, thus serving as shock absorbers. However, the findings of the present study suggest that this may be a defence mechanism, since elastic system fibres are degraded by MMPs even as they are synthesized. A greater proportion of MMP-7 and MMP-9-positive cells was detected in the posterior than in the anterior and intermediate bands of ADDwR and ADDwoR discs, demonstrating that disc degeneration begins in this area, as documented by Orhan and colleagues ${ }^{35}$.

The expression of these MMPs is regulated by several factors including a variety of cytokines (e.g. interleukin 1 and TNF- $\alpha$ ), ${ }^{45}$ which play an important role in TMJ ID pathogenesis and have indeed been demonstrated in synovial fluid of pathological TMJ. In contrast, tissue inhibitors of metalloproteinases (TIMPs) are matrixin inhibitors involved in the local control of MMP activities in tissues. A greater understanding of these processes could identify ways to curb MMP overproduction without compromising tissue remodelling.

The design of specific inhibitors for these MMPs is therefore an important objective. Such inhibitors would not only help to gain insights into the biological roles of MMPs, but

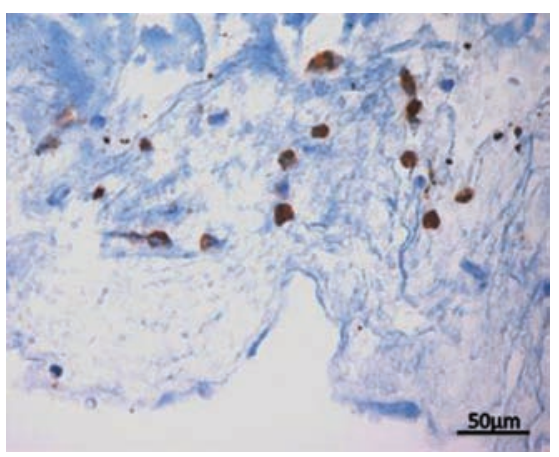

Figure 3. MMP-7 immunoexpression in synovium of a patient with TMJ ID and ADDwoR; magnification: 40x; scale bar: $50 \mu \mathrm{m}$. 
they would also aid in developing therapeutic interventions for diseases associated with abnormal ECM degradation, at least to slow down disease progression. Furthermore, MMP inhibitors such as sub-antimicrobial levels of tetracyclines or non-antimicrobial chemically modified tetracyclines could be beneficial in conditions involving MMP-mediated inflammatory tissue destruction, including TMJ ID. ${ }^{46}$ The findings of the present study contribute some novel information towards this purpose.

\section{References}

1. Verollet C, Charriere GM, Labrousse A, Cougoule C, Le Cabec V, MaridonneauParinil. Extracellular proteolysis in macrophage migration: losing grip for a breakthrough. Eur J Immunol 2011;41: 2805-13.

2. Loreto C, Musumeci G, Leonardi R. Chondrocyte-like apoptosis in temporomandibular joint disc internal derangement as a repair-limiting mechanism. An in vivo study. Histol Histopathol 2009; 24:293-8.

3. Westsson PL, Bronstein SL, Liedberg J. Internal derangement of the temporomandibular joint: morphologic description with correlation to joint function. Oral Surg Oral Med Oral Pathol 1985;59:323-31.

4. Lukaszewicz-Zajac M, Mroczko B, Szmitkowski M. Gastric cancer - The role of matrix metalloproteinases in tumor progression. Clin Chim Acta 2011;412:172530.

5. Yoncheva K, Momekov G. Antiangiogenic anticancer strategy based on nanoparticulate systems. Expert Opin Drug Deliv 2011;8:1041-56.

6. Detamore MS, Athanasiou KA. Structure and function of the temporomandibular joint disc: implications for tissue engineering. J Oral Maxillofac Surg 2003;61: 494-506.

7. Raffo D, Pontiggia 0, Simian M. Role of MMPs in metastatic dissemination: implications for therapeutic advances. Curr Pharm Biotechnol 2011;12:1937-47.

8. Krizkova S, Zitka 0, Masarik M, Adam V, Stiborova M, Eckschlager T, et al. Clinical importance of matrix metalloproteinases. Bratisl Lek Listy 2011;112:435-40.

9. Hua H, Li M, Luo T, Yin Y, Jiang Y. Matrix metalloproteinases in tumorigenesis: an evolving paradigm. Cell Mol Life Sci 2011; 68:3853-68.

10. Klein T, Bischoff R. Physiology and pathophysiology of matrix metalloproteases. Amino Acids 2011;41:271-90.

11. Leonardi R, Talic NF, Loreto C. MMP-13 (collagenase 3) immunolocalisation during initial orthodontic tooth movement in rats. Acta Histochem 2007;109:215-20.

12. Leonardi R, Caltabiano R, Loreto C. Collagenase-3 (MMP-13) is expressed in periapical lesions: an immunohistochemical study. Int Endod J 2005;38:297-301.

13. Agrawal SM, Yong VW. The many faces of EMMPRIN - roles in neuroinflammation. Biochim Biophys Acta 2011;1812:213-9.

14. Mizui T, Ishimaru J, Miyamoto K, Kurita K. Matrix metalloproteinase-2 in synovial lavage fluid of patients with disorders of the temporomandibular joint. Br J Oral Maxillofac Surg 2001;39:310-4.

15. Tanaka A, Kumagai S, Kawashiri S, Takatsuka S, Nakagawa K, Yamamoto E, et al. Expression of matrix metalloproteinase- 2 and -9 in synovial fluid of the temporomandibular joint accompanied by anterior disc displacement. J Oral Pathol Med 2001;30:59-64.

16. Kubota E, Kubota T, Matsumoto J, Shibata T, Murakami KI. Synovial fluid cytokines and proteinases as markers of temporomandibular joint disease. J Oral Maxillofac Surg 1998;56:192-8.

17. Leonardi R, Loreto C, Barbato E, Caltabiano R, Lombardo C, Musumeci G, et al. MMP-13 (collagenase 3) localization in human temporomandibular joint discs with internal derangement. Acta Histochem 2008;110:314-8.

18. Kudo Y, Iizuka S, Yoshida M, Tsunematsu T, Kondo T, Subarnbhesaj A, et al. Matrix metalloproteinase-13 (MMP-13) directly and indirectly promotes tumor angiogenesis. J Biol Chem 2012; 287:38716-28

19. Gu Z, Kaul M, Yan B, Kridel SJ, Cui J, Strongin A, Smith JW, Liddington RC, Lipton SA (2002) S-nitrosylation of matrix metalloproteinase: signaling pathway to neuronal cell death. Science 297:1186-1190

20. Caltabiano R, Leonardi R, Musumeci G, Bartoloni G, Rusu MC, Almeida LE, et al. Apoptosis in temporomandibular joint disc with internal derangement involves mitochondrial-dependent pathways. An in vivo study. Acta Odontol Scand 2012. In press.

21. Leonardi R, Almeida LE, Rusu M, Sicurezza E, Palazzo G, Loreto C. Tumor necrosis factor-related apoptosis-inducing ligand expression correlates to temporomandibular joint disk degeneration. J Craniofac Surg 2011;22:504-8.

22. Leonardi R, Migliore MR, Almeida LE, Trevilatto PC, Loreto C. Limited fatty infiltration due to apoptosis in human degenerated temporomandibular joint disks: an immunohistochemical study. J Craniofac Surg 2010;21:1508-11.

23. Loreto C, Almeida LE, Trevilatto P, 34. Srinivas R, Sorsa T, Tjaderhane L, Niemi E,
Leonardi R. Apoptosis in displaced temporomandibular joint disc with and without reduction: an immunohistochemical study. J Oral Pathol Med 2011;40:103-10.

24. Leonardi R, Almeida LE, Trevilatto PC, Loreto $\mathrm{C}$. Occurrence and regional distribution of TRAIL and DR5 on temporomandibular joint discs: comparison of disc derangement with and without reduction. Oral Surg Oral Med Oral Pathol Oral Radiol Endod 2010;109:244-51.

25. Loreto C, Galanti C, Almeida LE, Leonardi R, Pannone G, Musumeci G, et al. Expression and localization of aquaporin-1 in temporomandibular joint disc with internal derangement. J Oral Pathol Med 2012;41:642-47.

26. Leonardi R, Almeida LE, Loreto C. Lubricin immunohistochemical expression in human temporomandibular joint disc with internal derangement. J Oral Pathol Med 2011;40:587-92.

27. Sicurezza E. LC, Musumeci G., Almeida L.E., Rusu M.C., Grasso C., Leonardi R. Expression of -defensin 4 on temporomandibular joint discs with anterior displacement without reduction. J Craniomaxillofac Surg In press.

28. Musumeci G, Carnazza ML, Loreto C, Leonardi R, Loreto C. ${ }^{2}$-Defensin-4 (HBD4 ) is expressed in chondrocytes derived from normal and osteoarthritic cartilage encapsulated in PEGDA scaffold. Acta Histochem 2012; 114:805-812.

29. Loreto C, Lo Castro E, Musumeci G, Loreto F, Rapisarda G, Rezzani R, Castorina S, Rusu MC. Aquaporin 1 expression in human temporomandibular disc. Acta Histochem 2012; 114: 744-748.

30. Musumeci G, Leonardi R, Carnazza ML, Cardile V, Pichler K, Weinberg AM, Loreto C. Aquaporin 1 (AQP1) expression in experimentally induced osteoarthritis knee menisci: an in vivo and in vitro study. Tissue Cell 2013; in press.

31. Musumeci G, Loreto C, Carnazza ML, Cardile V, Leonardi R. Acute injury affects lubricin expression in knee menisci: an immunohistochemical study. Ann Anat 2012;pii:S0940-9602(12)00133-1 [Epub ahead of print].

32. Leonardi R, Rusu MC, Loreto C. Temporomandibular joint disc: a proposed histopathological degeneration grading score system. Histol Histopathol 2010;25: 1117-22.

33. Leonardi R, Loreto C, Barbato E, Polimeni A, Caltabiano R, Lo Muzio L. A histochemical survey of the human temporomandibular joint disc of patients with internal derangement without reduction. J Craniofac Surg 2007;18:1429-33.

open 2 access 
Raustia A, Pernu H, et al. Matrix metalloproteinases in mild and severe temporomandibular joint internal derangement synovial fluid. Oral Surg Oral Med Oral Pathol Oral Radiol Endod 2001;91:517-25.

35. Orhan K, Nishiyama H, Tadashi S, Murakami S, Furukawa S. Comparison of altered signal intensity, position, and morphology of the TMJ disc in MR images corrected for variations in surface coil sensitivity. Oral Surg Oral Med Oral Pathol Oral Radiol Endod 2006;101:515-22.

36. Pellicciari C, Malatesta M. Identifying pathological biomarkers: histochemistry still ranks high in the omics era. Eur $\mathrm{J}$ Histochem 2011;55:e42.

37. Kiga N. Histochemistry for studying structure and function of the articular disc of the human temporomandibular joint. Eur J Histochem 2012;56:e11.

38. Rodriguez-Lopez J, Perez-Pampin E, Gomez-Reino JJ, Gonzalez A. Regulatory polymorphisms in extracellular matrix protease genes and susceptibility to rheumatoid arthritis: a case-control study. Arthritis Res Ther 2006;8:R1.

39. Paulsen F, Pufe T, Conradi L, Varoga D, Tsokos M, Papendieck J, et al. Antimi crobial peptides are expressed and produced in healthy and inflamed human synovial membranes. J Pathol 2002;198: 36977.

40. Davidson RK, Waters JG, Kevorkian L, Darrah C, Cooper A, Donell ST, et al. Expression profiling of metalloproteinases and their inhibitors in synovium and cartilage. Arthritis Res Ther 2006;8:R124.

41. Pollanen PJ, Lehtimaki T, Mikkelsson J, Ilveskoski E, Kunnas T, Perola M, et al. Matrix metalloproteinase 3 and 9 gene promoter polymorphisms: joint action of two loci as a risk factor for coronary artery complicated plaques. Atherosclerosis 2005;180:73-8.

42. Ohta S, Imai K, Yamashita K, Matsumoto T, Azumano I, Okada Y. Expression of matrix metalloproteinase 7 (matrilysin) in human osteoarthritic cartilage. Lab Invest 1998;78:79-87.

43. Patterson BC, Sang QA. Angiostatin-converting enzyme activities of human matrilysin (MMP-7) and gelatinase B/type IV collagenase (MMP-9). J Biol Chem 1997;272:28823-5.

44. Leonardi R, Villari L, Bernasconi G, Caltabiano M. Histochemical study of the elastic fibers in pathologic human temporomandibular joint discs. J Oral Maxillofac Surg 2001;59:1186-92.

45. Ijima Y, Kobayashi M, Kubota E. Role of interleukin-1 in induction of matrix metalloproteinases synthesized by rat temporomandibular joint chondrocytes and disc cells. Eur J Oral Sci 2001;109:50-9.

46. Malemud CJ, Goldberg VM. Future directions for research and treatment of osteoarthritis. Front Biosci 1999;4:D76271. 